\title{
Contribución de las aplicaciones móviles a la Seguridad vial. Drive Smart como ejemplo de aplicación.
}

\author{
José Ignacio Lijarcio Cárcel \\ Investigador INTRAS. Universitat de València. España \\ Ana Martí-Belda Bertolín, Patricia Bosó Seguí, Consuelo López Osma, Cristina \\ Escamilla Robla, Raquel Puchades Villarreal \\ Investigadores INTRAS. Universitat de València.
}

\section{RESUMEN}

En los últimos años han proliferado las aplicaciones móviles que contribuyen, en diferentes aspectos, a mejorar la movilidad. En este contexto, se ha desarrollado la aplicación móvil Drive Smart, con el objetivo de analizar y mejorar el comportamiento del usuario al volante. A través de la utilización de dicha aplicación se obtiene un conjunto de datos que se transforman en un paquete de incidencias en la conducción, siendo éstas: velocidad, aceleración, frenada, paso por curva y anticipación al tráfico. Drive Smart analiza los eventos de tráfico registrados en el contexto en el que han ocurrido y como parte de una maniobra, más allá de la individualidad de un evento. Además, tiene la capacidad de asistir al conductor, dándole un informe posterior a su conducción que le sirva de autoformación y con consejos prácticos orientados a mejorar la conducta en temas particulares, mediante mensajes específicos de distintas tipologías (jurídico, eficiencia y sensibilizador). A través de este proyecto, se pretende demostrar la viabilidad y utilidad de los modernos mecanismos telemáticos en combinación con el estudio psicosociológico y las diversas técnicas de análisis estadístico de datos basados en Machine Learning. Los datos obtenidos de la aplicación móvil junto con datos sociodemográficos permiten obtener las principales variables de movilidad, además, pueden utilizarse para definir la base algorítmica en futuros estudios con mayor calado, teniendo una matriz estadística preliminar que permita generar procesos paralelos en el futuro.

\section{INTRODUCCIÓN}

La Seguridad vial es una disciplina que aborda todos los aspectos relacionados con la seguridad durante la conducción de vehículos. Por ello, el objetivo de una gran parte de especialistas en la materia es dotar a las personas que conducen de los conocimientos, aptitudes y herramientas adecuadas para aumentar la seguridad en la conducción y evitar los accidentes, así como minimizar los daños en caso de que éste se produzca. En este objetivo por conseguir aumentar dicha seguridad, las nuevas tecnologías juegan un papel importante. En los últimos años con el aumento del uso de teléfonos inteligentes o smartphones y las aplicaciones móviles, surgen nuevas herramientas para facilitar la tarea de conducción al propio usuario y que pueden suponer un elemento de valor en la movilidad actual.

Actualmente nuestra sociedad se encuentra constantemente conectada y en completa interacción con el medio que le rodea gracias en parte a la proliferación de las nuevas tecnologías. Esto mismo ocurre en el ámbito del tráfico, donde el usuario de la vía es cada vez más un "sujeto conectado" y que demanda una movilidad segura, eficiente y sostenible.

La concienciación del usuario hacia un tipo concreto de movilidad eficiente y segura es fruto de diversos aspectos. En primer lugar, el propio usuario ha aumentado su sensibilización 
frente a problemas como la contaminación, el consumo de combustible o la accidentalidad vial, resultado de ello es la constante disminución en las cifras de siniestralidad que se ha experimentado en los últimos años y la acusada demanda de modelos de conducción eficiente. Por otra parte, cada vez son más las empresas tecnológicas que dentro de sus departamentos de I+D, generan nuevas herramientas destinadas a la mejora de la conducción, lo cual es reforzado por las administraciones para conseguir sus objetivos de reducción de siniestralidad y aumento de eficiencia energética.

La citada proliferación en el uso de smartphones sigue un ascenso imparable que trae consigo una innumerable cantidad de aplicaciones móviles (app) con muy diversos usos o finalidades, contribuyendo, entre muchas otras cosas, a la movilidad. La movilidad es un aspecto vital en nuestro día a día. El hecho de desplazarse implica mucho más que un movimiento, puesto que a ese desplazamiento le sigue una actitud ante algo tan fundamental como la convivencia en la vía pública. Precisamente el hecho de convivir y compartir la vía exige por parte del conductor una conducción proactiva y que tiene a la base tanto actitudes como valores de respeto, solidaridad, tolerancia, y seguridad para el propio conductor y para el cada vez más valorado medio ambiente.

Es ampliamente conocida la existencia de los tres grandes factores que pueden influir en un accidente (factor humano, vía y entorno y factor vehículo) y el alto porcentaje de atribución del factor humano en la accidentalidad (70-90\%), porcentaje que podría ser todavía mayor teniendo en cuenta que el conductor, directa o indirectamente, tiene parte de responsabilidad en los otros dos factores. En este contexto nace Drive Smart, con el principal objetivo de generar una comunidad de conductores seguros, ya que se incide en la importancia del factor humano, a través del coaching vial personalizado, para cada conductor. Más allá del conocido consumo de alcohol, drogas, sueño etc., Drive Smart pretende actuar sobre las actitudes y los hábitos que hacen de nuestra conducción un continuo riesgo tanto para nosotros mismos como para los demás usuarios de la vía pública. Por ello, esta herramienta apuesta por el concepto de movilidad positiva y proactiva con todo lo que ello implica en la movilidad vial.

\section{QUÉ ES DRIVE SMART}

Drive Smart en un (app) gratuita la cual ha sido diseñada para analizar y mejorar el comportamiento al volante de los conductores. El análisis y la valoración de la conducción que realiza, se centra en el objetivo de conseguir que el usuario de esta herramienta sea un buen conductor, entendiéndolo como aquel que es eficiente en su consumo de combustible, responsable y respetuoso con el medio que le rodea y seguro en sus comportamientos al volante, evitando poner en riesgo la propia vida y la de los demás.

Drive Smart acompaña al conductor para monitorizar, interpretar y detectar sus conductas de riesgo en la conducción. El sistema evalúa y analiza las maniobras al volante: desde la reacción ante señales de tráfico y la circulación en rotondas hasta la toma de curvas urbanas, la frenada o la velocidad, entre otras. A través de una serie de datos y variables registradas por la app, Drive Smart aporta al conductor información específica sobre su conducción, de forma que progresivamente le ayuda a mejorar su conducta al volante.

Tras el análisis de estas variables se tutela y conciencia al usuario para que consiga los objetivos mencionados anteriormente, por lo que la aplicación no actúa únicamente como observador de la conducta, sino que favorece la mejoría de determinados comportamientos 
de riesgo. Por ello, es importante tener en cuenta las siguientes consideraciones:

- Drive Smart no hace un seguimiento a los conductores, no facilita datos privados y tampoco controla los movimientos o las localizaciones en las que se encuentran en cada momento.

- La privacidad y la garantía de confidencialidad de los datos del usuario es uno de los compromisos más firmes del equipo Drive Smart.

Con todo lo anterior, podemos decir que Drive Smart tiene la función principal de actuar como "coach de la conducción". El coaching es un método de trabajo mediante el cual, a través de un entrenador (coach, conseguimos obtener los mejores resultados de la persona, en las diferentes áreas en las que se esté trabajando. En nuestro caso, la app es el entrenador que a través de una serie de mensajes de reflexión y motivacionales, pretende mejorar la conducta al volante para que esta sea más segura y eficiente. Para ello se apela a la cognición, las emociones y los valores, mediante una serie de mensajes e informaciones adecuadas al caso concreto del conductor con el que se está trabajando.

\subsection{Funcionamiento de la aplicación.}

La instalación y funcionamiento de la aplicación es sencillo y la utilización continuada por parte del usuario elimina el posible sentimiento de "observación" que el conductor pueda percibir en un primer momento. Un aspecto de especial interés es el relacionado con la sincronización que se establece entre el coche y el smartphone, de forma que el registro de datos se realiza de forma automática al iniciar un trayecto. Con esto, se evitan los posibles problemas que pudiera generar una aplicación que necesite de la interacción directa con el usuario, aumentando en ese caso las distracciones al volante.

El registro de datos y variables se inicia al comenzar un trayecto y al finalizar el mismo, la app almacena los distintos eventos o incidencias que han ocurrido durante el recorrido para que el conductor pueda consultarlo en el momento que desee. Los eventos o incidencias registrados durante la conducción se clasifican en las siguientes dimensiones:

- Velocidad: conducción por encima o debajo de los límites indicados para esa vía.

- Aceleración: evaluación del proceso de aceleración y deceleración en un tramo de la vía como respuesta a los diferentes eventos del tráfico.

- Frenada: nivel de deceleración y detención del vehículo ante los diferentes eventos de la vía.

- Giro: maniobrabilidad y movilidad por la vía, derecha, izquierda, cambio de sentido.

- Anticipación al tráfico: proceso de reconocimiento y anticipación a la señalización vertical u horizontal.

Así pues mediante el análisis de los datos obtenidos, la app proporciona un feedback inmediato al usuario, nunca durante la conducción, informándole sobre los eventos anteriormente mencionados, la localización y momento en el que se han producido, así como su grado de severidad (peligrosidad).

Además, Drive Smart no actúa únicamente como una herramienta descriptiva de los 
acontecimientos potencialmente peligrosos en el tráfico, sino que como se comentaba en líneas anteriores, lleva a cabo una función de coaching que dota de especial valor a esta herramienta. Por tanto, la aplicación ofrece un feedback constante al usuario con el que analiza sus comportamientos al volante en cada uno de los eventos registrados y conciencia y tutela sobre sus hábitos en la conducción, a través de:

- Mensajes sobre las incidencias individuales ocurridas a lo largo de los recorridos en las distintas dimensiones (velocidad, aceleración, frenada, giro o anticipación al tráfico): este "pull de mensajes" contiene información legislativa, sensibilizadora sobre seguridad vial, información sobre conducción preventiva y eficiente, sobre el buen uso y mantenimiento de los componentes de seguridad pasiva y activa de los vehículos, así como de los principales factores y conductas de riesgo implícitos en el accidente. El contenido de los mensajes ha sido determinado en base a una amplia revisión bibliográfica sobre los conceptos de seguridad vial relacionados con el factor humano, y especialmente con aquellos factores que tienen una medición directa en la aplicación Drive Smart. A su vez, los mensajes son diferentes en función de distintas variables como:

- Evento registrado: velocidad, aceleración, giro, anticipación al tráfico y frenada.

- Severidad del evento: leve, moderado, severo.

- Lugar donde ocurre el evento: zona urbana o interurbana.

- Motivación del mensaje: mensajes con diferentes aproximaciones motivacionales dirigidas al sujeto (eficiencia energética/medioambiente, jurídico/legislativo y accidentalidad y sensibilización).

Con esta combinación de variables se consigue que los mensajes sean específicos y adaptados para las múltiples situaciones que pueden darse en el tráfico en combinación de los factores mencionados.

- Mensajes sobre las puntuaciones obtenidas: puntuación global sobre las dimensiones de cada viaje registrado, puntuación total en cada dimensión y puntuación total del trayecto.

- Informes mensuales: mensualmente, el usuario recibe informes sobre los resultados obtenidos a lo largo del mes. En estos informes se detallan las puntuaciones globales del mes, las dimensiones con mejores resultados (con el objetivo de reforzar estas conductas), las dimensiones con peores resultados (con el objetivo de plantear al usuario el reto de mejora), noticias adaptadas a las circunstancias del mes (mensajes sobre el verano, conducción en invierno, retenciones por salidas vacacionales...etc.) y que pueden enlazar al blog de Drive Smart.

- Mensajes con programación determinada en dos tipos: en primer lugar, mensajes con la finalidad de proporcionar consejos sobre seguridad vial a los usuarios de forma individual, y en segundo lugar, mensajes sobre el uso de la aplicación y estrategias para mejorar su utilización y aprovechamiento.

- Pit Stop: espacio destinado a ofrecer recomendaciones, retos, recompensas sobre diferentes temas de movilidad y seguridad vial, mitos o falsas creencias extendidos socialmente que tienen los usuarios.

Todos los mensajes que proporciona la aplicación han sido traducidos a 7 idiomas 
(castellano, inglés, alemán, francés, italiano, portugués y catalán) y siguen la misma estructura genérica en cuanto a longitud y número de caracteres. Sin embargo, algunos de ellos necesitan más espacio para poder elaborar ideas coherentes y completas, en estos casos, cuando tienen una longitud mayor, hay que accionar un botón ("leer más") para poder acceder al mensaje completo o a información adicional como la disponible en páginas web externas o al mismo blog de la página de Drive Smart si ya se han elaborado entradas relacionadas con el mensaje en cuestión.

En relación a la transculturalidad y la diferencia de horarios y épocas anuales en los distintos países en los que funciona la aplicación, la redacción de cada mensaje ha tenido en cuenta que los contenidos sean lo más neutros posible para poder ser difundidos en todos los países con independencia de normativas o estaciones del año distintas. Una vez desarrollada la programación completa de los mensajes en función del país donde se emitan, se podrán difundir los mensajes específicos y adaptados a cada lugar y momento (por ejemplo, un mayor número de mensajes de conducción en condiciones atmosféricas adversas en aquellos lugares donde el tiempo es desfavorable la mayor parte del año).

\section{3. ÁMBITOS DE APLICACIÓN DE LA APP}

Por sus características, Drive Smart puede encontrar numerosos ámbitos de utilización en el tráfico. Puesto que su objetivo es monitorizar, interpretar y detectar malos hábitos del conductor al volante, puede estar especialmente recomendado para todos los conductores en general, y muy especialmente para aquellos conductores noveles que necesitan obtener feedback en sus primeros momentos de contacto con el entorno del tráfico, de modo que adquieran un mayor nivel de confianza y seguridad en la carretera a través de la tutela de la aplicación, complementando toda la fase formativa previa que han debido cumplir previamente a la obtención del permiso o licencia de conducir.

Por otra parte, puede ser utilizada como una medida de apoyo a las aseguradoras para reducir la prima de aquellos que sean considerados como "buenos conductores" o "conductores seguros", ya que permite identificar parámetros y determinar el riesgo de sus conductores asegurados. En todo caso, resultados positivos pueden traducirse en importantes ventajas en las condiciones del seguro. Sin embargo, un resultado negativo no supone una mayor penalización para el asegurado.

Además de lo anterior, puesto que la utilización de la aplicación permite mejorar la conducción en dimensiones que pueden suponer una infracción o pérdida de puntos para el conductor, actúa como un elemento de intervención /prevención. Al hilo de esta función, cabe destacar el gran potencial que puede tener la aplicación en la reeducación de aquellos conductores multirreincidentes sobre los que sería interesante hacer un seguimiento más individualizado que permitiera comprobar si por ejemplo, cursos como los de sensibilización y reeducación vial, han sido efectivos y se traducen en una mejora en sus hábitos de conducción.

Por último, y especialmente importante teniendo en cuenta los problemas medioambientales a los que una sociedad como la nuestra está haciendo frente actualmente, Drive Smart puede servir como una interesante herramienta en el objetivo de conseguir una conducción eficiente. Dado que una gran mayoría de los conductores realiza una conducción que implica un alto consumo de combustible, el impacto ambiental podría reducirse drásticamente si dicho uso fuera el adecuado. No obstante, muchos usuarios no son conscientes de los comportamientos de conducción que tienen mayor impacto ambiental y económicamente, o 
no saben cómo mejorar esos hábitos. Drive Smart permite identificar dichos hábitos mediante el análisis de variables como las aceleraciones, las frenadas o la velocidad y actúa como un elemento de formación para conseguir que el conductor sea además de seguro, eficiente.

\section{PRIMEROS RESULTADOS}

Durante el desarrollo de la aplicación se realizaron distintos análisis que permitieron verificar la validez de la herramienta, entre los que cabe mencionar:

- Usabilidad de la app móvil: evaluación de la comprensión de la app en cada uno de sus apartados y la relación de la misma con el usuario final. Mejora de los menús de acceso, así como la estrategia en la presentación de la información.

- Base de maniobras telemáticas: evaluación de la definición y diseño de las variables/incidencias que registra la aplicación en base de las maniobras y conductas que realizan los conductores cuando manejan el vehículo.

- Mensajes de coaching: evaluación de la matriz de mensajes generados y su comprensibilidad por parte del usuario.

Por otra parte, tras la validación de los distintos aspectos de la app, se decidió analizar la posible existencia de tipologías de conductores. Para ello se realizó una categorización y análisis que permitiera definir distintos perfiles de usuarios en función de las incidencias registradas y de variables sociodemográficas recogidas de forma voluntaria y anónima. Dichos análisis corresponden una primera fase de pilotaje en el objetivo de categorizar a los conductores y establecer su perfil sociodemográfico y el riesgo potencial del usuario. Por ello se analizaron las siguientes variables:

- Eventos e incidentes registrados durante la conducción

- Perfil sociodemográfico de los conductores

- Percepción de la propia tarea de conducción

\subsection{Resultados de eventos e incidentes}

En una primera fase de la investigación, se analizaron los incidentes registrados por 2.729 usuarios de Drive Smart en los meses de mayo, junio, julio y agosto. Se realizó un análisis para comparar las medias obtenidas en cada uno de los eventos que registra la app (aceleración, frenada, velocidad, giro y anticipación al tráfico) donde los resultados mostraron que, respecto a los meses anteriores, en agosto los incidentes de aceleración disminuyeron un $6 \%$, los correspondientes a frenadas disminuyeron un $1 \%$, los incidentes relacionados con giros o curvas disminuyeron un $6 \%$, del mismo modo que los de velocidad (6\%) y los incidentes de anticipación al tráfico se redujeron en un 33\%.

Teniendo en cuenta las puntuaciones globales obtenidas durante los meses del periodo de estudio, se establecieron dos grupos de conductores claramente diferenciados por ser los ubicados en los extremos, es decir, los conductores con mejores y peores puntuaciones del total de la muestra. Los análisis mostraron que en el caso de los conductores con las más altas puntuaciones, la velocidad media de sus trayectos disminuyó en un $2 \%$ tras 4 meses de uso de la aplicación, mientras que en el caso de los conductores con puntuaciones globales más bajas, la velocidad media descendió en un 3\%, y los eventos de velocidad un $9 \%$. 


\subsection{Perfil sociodemográfico y percepción de la tarea de conducción}

En una segunda fase, se contó con una muestra de 90 usuarios de la aplicación, que de forma voluntaria y anónima facilitaron una serie de datos sociodemográficos y contestaron un conjunto de preguntas sobre la percepción que tenían sobre su propia tarea de conducción.

La edad de los usuarios que participaban en la investigación iba de los 18 a los 65 años $(M=33,73 ; D T=8,86)$. La distribución en rangos de edad mostró un mayor porcentaje de usuarios de 35 a 49 años (40,9\%), seguido del grupo de 25 a 34 años $(36,4 \%$ ) y 18 a 24 años $(18,2 \%)$. En cuanto al sexo, el $81,8 \%$ de los usuarios fueron hombres y el 18,2\% fueron mujeres. Por su parte, el nivel de estudios de los usuarios participantes fue mayoritariamente de titulados superiores $(38,1 \%)$ y bachillerato o equivalente $(38,1 \%)$. El 77,3\% afirmó estar trabajando y tres de cada diez conductores informó que realizaba trayectos acompañado de menores al menos tres veces por semana.

La mayoría de los usuarios utilizaba un turismo durante la investigación $(81,8 \%)$ y afirmó recorrer entre 10.000 y $30.000 \mathrm{~km} / \mathrm{año.}$

En cuanto a la pérdida de puntos y la accidentalidad, el $77 \%$ de los participantes afirmó no haber perdido ningún punto en su permiso. Porcentaje similar al obtenido cuando se les planteó la cuestión de los accidentes sufridos en los últimos 5 años, donde el 77,3\% de los participantes afirmó no haber sufrido ningún accidente.

Por otra parte, se realizó un análisis para hallar posibles relaciones entre la habilidad percibida por los usuarios y las puntuaciones objetivas registradas por Drive Smart en los cinco eventos que contempla la aplicación. El objetivo fue averiguar si la autopercepción que tenía el conductor sobre su conducción (medida subjetiva) se correspondía con los resultados obtenidos a través de la evaluación de la app, (medida objetiva).

Así, en cuanto a la aceleración, se halló una relación significativa $(p<0,05)$, entre la habilidad informada por los sujetos y la propia puntuación de la app, por lo que se pudo afirmar que aquellos usuarios que se consideran más hábiles respecto a sus aceleraciones, obtuvieron también puntuaciones más altas en el registro objetivo de este evento.

Por lo que respecta a los giros o pasos por curva, se obtuvo una relación significativa $(p<0,05)$, que indicó que a medida que los sujetos se perciben como mejores conductores en sus giros, obtienen también puntuaciones superiores en los registros que realiza la app. La misma tendencia siguió el análisis sobre la variable velocidad $(p<0,05)$, donde los sujetos que se perciben como más respetuosos hacia los límites de velocidad establecidos, son también los que obtienen mejores puntuaciones respecto a este aspecto de la conducción.

Con todo, los resultados permitieron concluir que existe una relación entre la percepción de habilidad de los participantes y su ejecución real de la tarea de conducir. Esto ofrece una interesante lectura de los resultados ya que, parecen indicar que existe una correspondencia entre la dimensión más subjetiva de la conducción, y la más objetiva. Éste es un aspecto destacable dada la frecuente distorsión entre la percepción que los conductores tienen sobre su tarea y la realidad posterior. Tanto es así, que uno de los problemas más frecuentes en el tráfico es la sobrevaloración de las propias habilidades y capacidades, aspecto que parece no afectar a los sujetos de la presente investigación. 


\section{CONCLUSIONES}

La nueva generación de herramientas tecnológicas por parte de las empresas está motivando tanto a las administraciones, entidades y usuarios al uso de aplicaciones que faciliten la movilidad y la conducción, tanto en su aspecto de eficiencia energética como de reducción de siniestralidad vial. Ante este escenario nace Drive-Smart, una aplicación que monitoriza y evalúa al conductor a través del análisis de cinco variables; aceleración, frenada, giro, anticipación al tráfico y velocidad. Con los resultados obtenidos en cada una de estas variables la aplicación genera un paquete de mensajes que actúa de "coach vial" al objeto de tutelar y mejorar los resultados en las variables mencionadas anteriormente. Los mensajes y feedback que obtiene el sujeto se realiza por diferentes vías: mensajes sobre los eventos registrados o incidencias, informe mensual con un resumen de las puntuaciones obtenidas en las cinco variables y retos de mejora, mensajes genéricos sobre seguridad vial y mejora de usabilidad de la aplicación y Pit Stop, planteamiento de retos para conseguir puntos canjeables por recompensas, informaciones y actividades lúdicas.

Tras la validación de los diferentes parámetros de la aplicación y la evaluación sobre la comprensión y el entendimiento por parte del usuario de los mensajes de coaching, se diseñó un estudio donde se permitiera establecer el perfil de los usuarios de Drive Smart. Además, dicho estudio tiene como objetivo categorizar a los conductores y establecer su perfil sociodemográfico y el riesgo potencial del usuario. Tras una primera fase de análisis, se comprobó que después de la utilización de la herramienta por parte de un grupo de usuarios, los incidentes de aceleración disminuyeron un $6 \%$, las frenadas disminuyeron un $1 \%$, los giros o curvas bruscos disminuyeron un $6 \%$, del mismo modo que los excesos de velocidad (6\%) y los incidentes de anticipación al tráfico (33\%). en una segunda fase, se analizó el perfil sociodemográfico del usuario y su percepción de riesgo. Los resultados obtenidos mostraron que los usuarios tenían una percepción de riesgo similar a la registrada objetivamente mediante la aplicación, lo que corrobora la credibilidad de la aplicación frente al registro de eventos o situaciones en la vía y ofrece una visión interesante de la percepción subjetiva de riesgo por parte del usuario y que normalmente no se corresponde con el riesgo objetivo o real que hay en una situación o evento de tráfico.

Actualmente la aplicación continúa en proceso de estudio, desarrollo y mejora a través del feedback que ofrecen los usuarios, tanto en la eficacia de la medición de las dimensiones, como en la mejora de los mensajes de coaching vial para conseguir el cambio de actitudes en los conductores. El contexto tecnológico actual favorece y mejora el uso de este tipo de herramientas con el objetivo de conseguir una movilidad más eficiente y segura, por lo que es importante continuar trabajando en estas líneas de investigación desde la seguridad vial y el transporte.

\section{AGRADECIMIENTOS}

Este proyecto fue realizado gracias a la financiación del CDTI a la compañía DRIVESMART, junto con el Instituto Universitario de Tráfico y Seguridad Vial de la Universidad de València. 